\title{
Effect of the retention ring-assisted continuous application of riboflavin in pulsed-light accelerated corneal collagen cross-linking on the progression of keratoconus
}

\author{
Chung Young $\mathrm{Kim}^{1}$ and Mee Kum Kim ${ }^{1,2^{*}}$ [D
}

\begin{abstract}
Background: To investigate the efficacy and safety of the retention ring-assisted continuous application of $0.1 \%$ riboflavin in pulsed-light accelerated corneal collagen cross-linking on the progression of keratoconus.

Methods: The medical records of 20 eyes of 18 patients with progressive keratoconus who received collagen crosslinking at Seoul National University Hospital were retrospectively reviewed. Isotonic $0.1 \%$ riboflavin was continuously applied for 10 min using an 8.0-mm retention ring before the irradiation and accelerated cross-linking was applied with 30-mW pulsed-ultraviolet light at a wavelength $365 \mathrm{~nm}$ for eight minutes $\left(1 \mathrm{~s}\right.$ on/1 s off; $30 \mathrm{~mW} / \mathrm{cm}^{2}$, cumulative dose of $7.2 \mathrm{~J} / \mathrm{cm}^{2}$ ) without further intermittent application of riboflavin. Visual acuity, refractive error, topographic index, corneal thickness, and endothelial cell density were evaluated before the operation and at 1, 3, 6, and 12 months.

Results: The best corrected visual acuity in logMAR improved from preoperative 0.43 to 0.17 in 12 months ( $p=0.050$ ). Maximum keratometry decreased from 51.8 D to 50.4 D at 6 months ( $p=0.015)$ and $50.1 \mathrm{D}$ at 12 months $(p=0.0003)$. Astigmatism decreased from preoperative $5.5 \mathrm{D}$ to $4.1 \mathrm{D}$ at 12 months $(p<0.0001)$. Thinnest corneal thickness decreased at three and 6 months but recovered in 12 months ( $p>0.05)$. Endothelial cell density decreased at postoperative 1 month ( $p=0.02$ ) but gradually recovered in 12 months $(p>0.05)$.

Conclusions: Retention ring-assisted continuous application of riboflavin for 10 minutes in pulsed-light accelerated crosslinking is a comparably safe and effective treatment for halting the progression of keratoconus in 12 months when compared to outcomes of the standard Dresden protocol shown in previous reports.
\end{abstract}

Keywords: Cornea, Keratoconus, Corneal cross-linking (CXL), Accelerated cross-linking, Pulsed-light, Retention ring, Riboflavin

\section{Background}

Keratoconus is an ectatic corneal disorder characterized by progressive thinning and protrusion, accompanied by irregular astigmatism and myopic progression, thereby deteriorating visual function. The conventional standardized treatment is keratoplasty in advanced keratoconus [1].

\footnotetext{
* Correspondence: kmk9@snu.ac.kr

'Department of Ophthalmology, Seoul National University Hospital, 101 Daehak-ro, Jongno-gu, Seoul 110-744, South Korea

${ }^{2}$ Laboratory of Ocular Regenerative Medicine and Immunology, Biomedical Research Institute, Seoul National University Hospital, 101 Daehak-ro, Jongno-gu, Seoul 110-744, South Korea
}

Corneal collagen cross-linking has recently been investigated to intervene in progression of moderate keratoconus. In corneal cross-linking (CXL), riboflavin (vitamin B2) acts as a photosensitizer. When it is combined with ultraviolet A (UV-A) of 365-nm wavelength, oxygen radicals are produced via photochemical reaction. Oxygen radicals can introduce interfibrillar and intrafibrillar covalent bonds among collagen fibrils [2,3]. Such chemical bonds can increase the corneal rigidity and its resistance to enzymatic degradation of keratoconic cornea [4],

(c) The Author(s). 2019 Open Access This article is distributed under the terms of the Creative Commons Attribution 4.0 International License (http://creativecommons.org/licenses/by/4.0/), which permits unrestricted use, distribution, and 
thereby restoring the stability of the cornea and halting the progression of keratoconus.

In the standard Dresden protocol, cornea is deepithelialized using alcohol $20 \%$ solution and soaked in isotonic $0.1 \%$ riboflavin with $20.0 \%$ dextran solution for $30 \mathrm{~min}$. The cornea is then irradiated with UV-A of $3 \mathrm{~mW} / \mathrm{cm} 2$ for 30 min (total energy $5.4 \mathrm{~mJ} / \mathrm{cm} 2$ ). Riboflavin is intermittently applied before and during the irradiation [5]. This protocol can effectively stabilize the cornea of progressive keratoconus in 10 years [6]. However, it is considered time-consuming by some clinicians.

An accelerated cross-linking protocol was then introduced to shorten the procedure. It applies UV-A of greater intensity for a shorter irradiation time based on the Bunsen-Roscoe law of reciprocity [7]. According to the theory, total energy delivered to the cornea remains identical. Its biological effect is proportional to the energy delivered. Surgical procedure of accelerated protocol is basically the same as the standard Dresden protocol except two aspects; the intensity (from 9 to $30 \mathrm{~mW} / \mathrm{cm}^{2}$ ) and irradiation time of UV-A (from 3 to $15 \mathrm{~min}$ ) [8-16]. Many studies have reported that clinical results of the accelerated protocol are comparable to those of the standard Dresden protocol. However, combination of intensity and irradiation time is diverse. It has not been standardized yet. Furthermore, recent meta-analyses have shown that the standard Dresden protocol has better treatment efficacy in depth of demarcation line and change of keratometric values than the accelerated protocol $[15,16]$.

A study on photochemical kinetics of cross-linking has shown that continuous UV-A irradiation can induce rapid depletion of oxygen in riboflavin applied corneal tissue and that turning the UV off can replenish oxygen level [17]. Photodynamic reaction between riboflavin molecules and UV-A can induce release of reactive oxygen species (ROS) and introduces cross-linking in collagen fibers. According to the kinetics model, pulsing the UV-A irradiation theoretically can restart the photodynamic reaction and maintain ROS concentration in the cornea [17]. Based on these findings, pulsed-light accelerated cross-linking had been adopted to enhance treatment efficacy. It has been reported to be as effective as the standard Dresden protocol [18-20].

So far, there is no standardized method for riboflavin application in the accelerated protocol. The protocol provided by the manufacturer (Avedro. Inc., Waltham, MA, USA) recommends intermittent application of isotonic riboflavin every 2 minutes for a total of 10 minutes. Recent studies have reported different clinical efficacies with various application time (from 10 to $30 \mathrm{~min}$ ) and intervals (2 to 5 minutes) [9-12, 19]. Shorter application time (every 2 minutes for $10 \mathrm{~min}$ ) seems to have lower efficacy, even in pulsed-light accelerated protocol, than the standard protocol [12]. In addition, our group has recently demonstrated possible damage to the limbal stem-cell area by exposure to riboflavin during cross-linking [21]. Thus, it is necessary to protect the corneal limbal area and prevent it from exposure to riboflavin and UV.

To enhance the efficacy of cross-linking in progressive keratoconus and add limbal protection, our group adopted pulsed-light cross-linking protocol with two modifications. First, we introduced a retention ring for the application of riboflavin in order to apply riboflavin continuously $(10 \mathrm{~min})$ for better penetration into the cornea while enhancing the efficacy and reducing exposure of limbal area to riboflavin. Second, we applied a trephined silicone hydrogel bandage contact lens on limbal area during irradiation of UV-A to protect it from toxic effect of UV [21]. UV-A was irradiated in a pulsatile mode ( $1 \mathrm{~s}$ on and off) for a total of $8 \mathrm{~min}$ with total energy of $7.2 \mathrm{~J} / \mathrm{cm}^{2}$ as described in a previous report [18]. We hypothesized that this novel method of retention ring-assisted continuous application of $0.1 \%$ riboflavin for 10 minutes in pulsed-light accelerated cross-linking might have better or comparable efficacy and safety profile for halting the progression of keratoconus than other pulsed modes, accelerated cross-linking protocol, or standard Dresden protocol described in previous reports.

\section{Methods \\ Patients}

This study was approved by the Institutional Review Board of Seoul National University College of Medicine (IRB No. 1807-139-961, Seoul, South Korea) and adhered to the Declaration of Helsinki. It included 20 eyes of 18 patients with progressive keratoconus who received CXL at a single institution (Seoul National University Hospital) by a single surgeon (M.K. Kim). The medical records of the 18 patients (14 male and 4 female patients) were retrospectively reviewed. The mean age was $28.0 \pm 7.3$ (range 17-43) years, and all patients were treated with the same protocol.

\section{Inclusion and exclusion criteria}

The patients who were diagnosed with progressive keratoconus and underwent accelerated CXL were included in the study. Progression was confirmed by an increase of maximum keratometry $\left(K_{\max }\right)$ of more than 1.5 diopters (D) / year in serial topography. Those with preoperative $K_{\max }$ greater than $60 \mathrm{D}$ or central corneal thickness (CCT) less than $400 \mu \mathrm{m}$ or thinnest corneal thickness (TCT) less than $390 \mu \mathrm{m}$ were excluded. Patients combined with other ocular surface diseases were also excluded.

\section{Clinical evaluation}

Preoperative and postoperative examinations included best-corrected visual acuities (BCVA) as a logarithm of the minimum angle of resolution (logMAR), refractive errors by Auto Kerato-Refractometer (KR-8900, Topcon, 
Tokyo, Japan), keratometric values including maximum $\left(K_{\max }\right)$, minimum $\left(K_{\min }\right)$, average $\left(K_{\mathrm{avg}}\right)$, and $3 \mathrm{~mm}$ and $5 \mathrm{~mm}$ irregular index by topography (ORBSCAN IIz, Technolas Perfect Vision GmbH, München, Germany). Corneal thickness (CT) was measured by anterior segment optical coherence tomography (AS-OCT, Visante OCT; Carl Zeiss Meditec, Dublin, CA). Noncontact specular microscopy (SP-8800, Konan, Hyogo, Japan) was used to measure endothelial cell density. Preoperative measurements were compared with the postoperative measurements at $1,3,6,12$ months.

\section{Surgical procedure}

Eyes were anesthetized with topical $1 \%$ proparacaine (Alcaine; Alcon, Fort Worth, TX) and the central 9.0-mm corneal epithelium was peeled off using a crescent knife (Beaver; Beaver-Visitec, Waltham, MA). Intraoperative pachymetry (Pocket II; Quantel Medical, Bozeman, MT) was performed to make sure that the corneal thickness was greater than $325 \mu \mathrm{m}$. A retention ring $8.0 \mathrm{~mm}$ in diameter (Frimen, Inc., Jiangsu, China) was applied on the epi-off corneal surface, and $0.1 \%$ isotonic riboflavin (Vibex Rapid, Avedro, Inc., Waltham, MA, USA) with dextran-free hydroxypropyl methylcellulose (HPMC; VibeX Rapid, Avedro, Waltham, MA) was continuously applied for 10 minutes within the retention ring (Additional file 1: Figure S1 A). Then, a trephined (inner diameter, $8.5 \mathrm{~mm}$ ) silicone hydrogel bandage contact lens (ACUVUE OASYS; Johnson \& Johnson Vision Care, Jacksonville, FL) was applied to cover the limbus from UV irradiation (Additional file 1:Figure S1 $B$ and C). Pulsing (1 s. on/off) $30 \mathrm{~mW} / \mathrm{cm}^{2}$ intensity of 365-nm wavelength UV-A (Avedro, Waltham, MA) was irradiated for 8 minutes, resulting in a cumulative dose of $7.2 \mathrm{~J} / \mathrm{cm}^{2}$. During the irradiation, riboflavin was not applied. After irradiation, the corneal surface was irrigated with a balanced salt solution, and the silicone hydrogel bandage contact lens was applied. The bandage contact lens was maintained for 7 days with topical $0.5 \%$ moxifloxacin and $1 \%$ prednisolone four times a day.

\section{Statistical analysis}

Statistical analysis was performed using Prism 5 software (Graphpad Software, Inc., San Diego, CA). The two-tailed Student's $t$ test was used to compare each of the baseline parameters with the following measurement. The data are presented with mean value \pm mean standard deviation (SD), and statistical significance was verified if $p<0.05$.

\section{Results}

This study analyzed a total of 20 eyes of 18 patients over a mean postoperative follow-up of $12.7 \pm 1.5$ months. The demographics and baseline characteristics of the study group are shown in Table 1 . The mean age at the time of the procedure was $28.0 \pm 7.3$ years. Mean depth of the demarcation line was $281.0 \pm 39.9 \mu \mathrm{m}$ (minimum $210 \mu \mathrm{m}$ to maximum $380.0 \mu \mathrm{m}$ ) (Additional file 2: Figure S2, Table 1). The penetration depth (demarcation line) in our study is comparable to or deeper than that in other studies on standard Dresden protocol or accelerated cross-linking protocols (Table 2).

The best corrected visual acuity gradually improved from preoperative $0.43 \pm 0.43 \log$ MAR to $0.17 \pm 0.16 \log$ MAR at 12 months with a marginal significance $(p=0.050)$ (Fig. 1a) and uncorrected visual acuity also improved, from $0.81 \pm$ $0.38 \log$ MAR to $0.49 \pm 0.50 \log$ MAR at 12 months, although it was not statistically significant $(p=0.635)$ (Fig. $1 b)$. Refractive errors decreased from $-7.40 \pm 2.80 \mathrm{D}$ to $6.25 \pm 3.11 \mathrm{D}$ in spherical $(p=0.245),-4.81 \pm 1.36 \mathrm{D}$ to $4.00 \pm 2.07 \mathrm{D}$ in cylinder $(p=0.003)$ and $-9.81 \pm 2.93 \mathrm{D}$ to $-8.25 \pm 2.98 \mathrm{D}$ in spherical equivalent $(p=0.075)$ after 12 months, and remained stable thereafter respectively) (Fig. $1 \mathrm{c}$ and d). Cylindrical reduction was a statistically significant with this modified protocol.

Keratometric values were delineated as $K_{\max }$ (maximum), $K_{\min }$ (minimum), and $K_{\text {avg }}$ (average). $K_{\max }$ (Fig. 2a) significantly decreased from $51.85 \pm 4.11 \mathrm{D}$ to $50.36 \pm 3.55 \mathrm{D}$ at 6 months $(p=0.015)$ and $50.11 \pm 3.62 \mathrm{D}$ at 12 months $(p=$ 0.0003). $K_{\min }$ (Fig. $2 \mathrm{~b}$ ) decreased, from $46.40 \pm 2.19 \mathrm{D}$ to $45.99 \pm 2.27 \mathrm{D}$ at 12 months $(p=0.113)$ although it was insignificant. $K_{\text {avg }}$ (Fig. 2c) also significanly decreased from $49.12 \pm 2.95 \mathrm{D}$ to $48.55 \pm 2.79 \mathrm{D}$ at 6 months $(p=0.045)$ and $48.05 \pm 2.69 \mathrm{D}(p=0.0028)$ at 12 months. Topographic astigmatism was significantly reduced from preoperative $5.45 \pm 2.90 \mathrm{D}$ to $4.23 \pm 2.27 \mathrm{D}$ at 6 months $(p=0.006)$ and $4.10 \pm 2.75 \mathrm{D}$ at 12 months $(p<0.0001)$ (Fig. $2 \mathrm{~d})$. Irregular index at $3 \mathrm{~mm}$ and $5 \mathrm{~mm}$ decreased from preoperative 6.27 $\pm 2.16 \mathrm{D}$ to $5.06 \pm 1.53 \mathrm{D}(p=0.006)$ and $6.11 \pm 1.82 \mathrm{D}$ to $5.36 \pm 1.38 \mathrm{D}(p=0.017)$, and the reductions were statistically significant at 12 months. (Fig. 2e and f).

Central corneal thickness showed a significant decrease, from $495.9 \pm 36.1 \mu \mathrm{m}$ to $484.5 \pm 41.4 \mu \mathrm{m}$ in 12 months $(p$ $=0.020)$ (Fig. 3a). Thinnest corneal thickness was temporarily decreased at 6 months $(p=0.0025)$, but eventually recovered to $460.5 \pm 38.3 \mu \mathrm{m}$ in 12 months, resulting in no statistical difference compared with the preoperative level ( $p>0.05$, pre-operation vs 12 mo.) (Fig. 3b).

Endothelial cell density significantly decreased, from preoperative $2693 \pm 223$ cells $/ \mathrm{mm}^{2}$ to $2434 \pm 460$ cells/ $\mathrm{mm}^{2}$ at 1 month $(p=0.018)$, but gradually recovered back in 12 months $\left(2621 \pm 471\right.$ cells $\left./ \mathrm{mm}^{2}\right)$ without any significant change compared with preoperative level or 3 months ( $p=0.325$, preop vs 12 mo., $p=0.08,3$ mo. vs 12 mo.) (Fig. 3c).

There was a single case of delayed wound healing, which spontaneously resolved in 14 days with conservative treatments using preservative artificial tear; otherwise, there were no serious complications related to the procedure. 
Table 1 Baseline characteristics of the patients who underwent pulsed accelerated cross-linking in this study

\begin{tabular}{|c|c|}
\hline \multicolumn{2}{|l|}{ Demographic factors } \\
\hline Eyes & 20 eyes of 18 patients \\
\hline Sex & 14 male $(77.7 \%), 4$ female $(22.3 \%)$ \\
\hline Age (year) & $28.0 \pm 7.3$ \\
\hline Systemic disease / Atopy & $0(0 \%) / 2(11 \%)$ \\
\hline FU (month) & $61.3 \pm 53.6$ \\
\hline POD (month) & $12.8 \pm 1.0$ \\
\hline BCVA (logMAR) & $0.43 \pm 0.43$ \\
\hline UCVA (logMAR) & $0.81 \pm 0.38$ \\
\hline \multicolumn{2}{|l|}{ Refractive error } \\
\hline Spherical (diopter) & $-7.40 \pm 2.80$ \\
\hline Cylinder (diopter) & $-4.81 \pm 1.36$ \\
\hline SE (diopter) & $-9.81 \pm 2.93$ \\
\hline \multicolumn{2}{|l|}{ Topography } \\
\hline Kmax (diopter) & $52.20 \pm 4.32$ \\
\hline Kmin (diopter) & $46.50 \pm 2.18$ \\
\hline Kavg (diopter) & $49.35 \pm 3.06$ \\
\hline Astigmatism & $5.71 \pm 3.07$ \\
\hline $\mathrm{IR}, 3 \mathrm{~mm}$ & $6.38 \pm 2.17$ \\
\hline $\mathrm{IR}, 5 \mathrm{~mm}$ & $6.23 \pm 1.86$ \\
\hline \multicolumn{2}{|l|}{ AS-OCT } \\
\hline$C C T(\mu \mathrm{m})$ & $492.1 \pm 39.1$ \\
\hline $\mathrm{TCT}(\mu \mathrm{m})$ & $466.6 \pm 37.1$ \\
\hline ECD (cells/ mm²) & $2731 \pm 279.5$ \\
\hline $\begin{array}{l}\text { Depth of demarcation line } \\
\text { after } 1 \mathrm{mo} \text { of cross-linking }(\mu \mathrm{m})\end{array}$ & $281.0 \pm 39.9$ \\
\hline
\end{tabular}

Values are presented as mean $\pm S D$, FU, follow up; POD, postoperative date; $B C V A$, best-corrected visual acuity; UCVA, uncorrected visual acuity; logMAR, logarithm of minimum angle of resolution; $\mathrm{SE}$, spherical equivalent; IR, irregular astigmatism; CCT, central corneal thickness; TCT, thinnest corneal thickness; AS-OCT, anterior segment optical coherence tomography; ECD, endothelial cell density

\section{Discussion}

In this study, we report that retention ring-assisted continuous application of riboflavin for 10 minutes in pulsed-light accelerated cross-linking is as effective as standard Dresden protocol or more effective than other pulsed-light accelerated cross-linking protocols for halting the progression of keratoconus in 12 months. With regard to safety, endothelial cell density changes in 12 months of this protocol are comparable to outcomes described in previous reports (Table 2).

To date, there is no consensus on a standardized protocol for accelerated cross-linking comparable to the standard Dresden protocol, which has been documented to be effective in treating progressive keratoconus by improving corrected distance visual acuity, decreasing keratometric values, and preserving endothelial cells in a ten-year follow-up study [6]. To improve efficacy, a pulsed-light accelerated protocol was first introduced, showing better functional outcomes in 1 year by optimizing intraoperative oxygen availability than the standard Dresden protocol [18]. It also showed effectiveness in a two-year follow-up [20]. Nevertheless, recent meta-analysis studies generally indicate that the standard Dresden protocol has better efficacy than for the accelerated protocol with a deeper demarcation line $[15,16]$. However, protocol modification is still required to improve outcomes of the accelerated protocol. We hypothesized that continuous application of riboflavin using a retention ring might induce deeper penetration of riboflavin and increase the availability of riboflavin in corneal tissue, thus enhancing the efficacy of treatment.

To shorten the procedure time without reducing its efficacy, we aimed to modify the application method of riboflavin by continuously using a retention ring before irradiation. The overall clinical effect of our modified protocol on visual acuity, topographic parameters and demarcation line depth was comparable to that of the standard Dresden protocol or better than other studies with accelerated cross-linking (Table 2). The depth of demarcation line in this study was the greatest $(281 \mu \mathrm{m})$ among pulsed-light accelerated protocols reported (202 $\sim 280 \mu \mathrm{m})$. It was comparable with other continuous accelerated protocols $(184 \sim 317 \mu \mathrm{m})$ or the standard Dresden protocol $(280 \sim 442 \mu \mathrm{m})$. The mean visual improvement was $-0.25 \operatorname{logMAR}$ in our study, which was better than the outcome in the accelerated protocol $(-0.01 \sim-0.14 \operatorname{logMAR})$ and the standard Dresden protocol $(-0.05 \sim-0.13 \operatorname{logMAR})$. The mean reduction of maximum keratometry was $-1.54 \mathrm{D}$ in our study. It was greater that of other accelerated protocols $(-0.3 \mathrm{D}$ $\sim-1.31 \mathrm{D})$ and comparable to that of the standard Dresden protocol $(-0.76 \mathrm{D} \sim-1.80 \mathrm{D})$ (Table 2). Therefore, our modified ten-minute continuous application of riboflavin in a pulsed-light accelerated protocol has better efficacy than other accelerated protocols. It is as efficient as the standard Dresden protocol in halting the progression of keratoconus.

Because of the possibility of limbal stem cell damage during riboflavin application and UV irradiation [21], modification of the previous protocol as essential. In our in vivo experiments [21], a significantly greater decrease of $\mathrm{p} 63^{+}$stem cells was observed when riboflavin was dropped on the whole corneal surface in accelerated cross-linking when compared to the protocol using the retention ring to confine riboflavin to only the central cornea. We believe that protecting the limbal stem cells by minimizing their exposure to riboflavin and UV is important, given their crucial role in regeneration of cells. Further experimental studies on concentrationand duration-dependent toxicity of riboflavin are needed to elucidate cellular injury and standardize the protocol. 





A

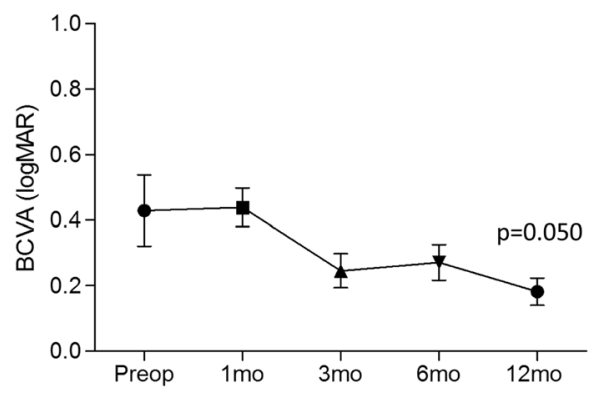

C

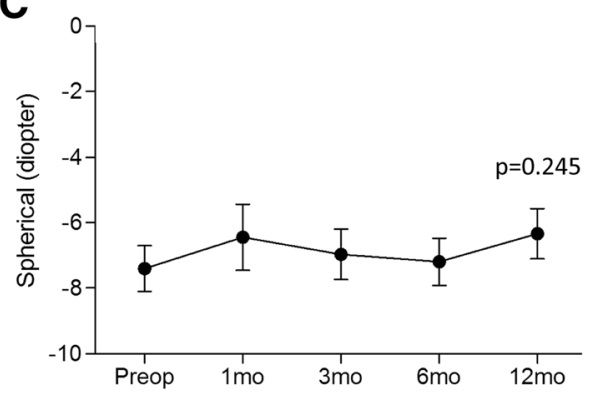

B

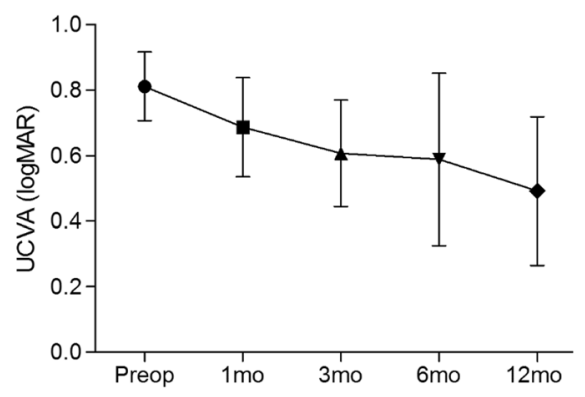

D

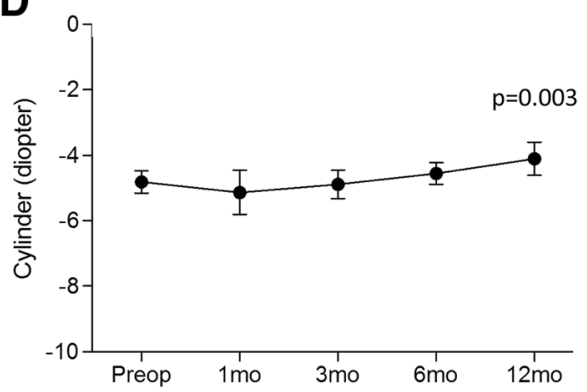

Fig. 1 Changes of best corrected visual acuity (BCVA, logMAR) (a), uncorrected visual acuity (UCVA, logMAR) (b) and spherical (c), cylindrical (d) refractive errors (diopter) over time following modified pulsed-light accelerated cross-linking

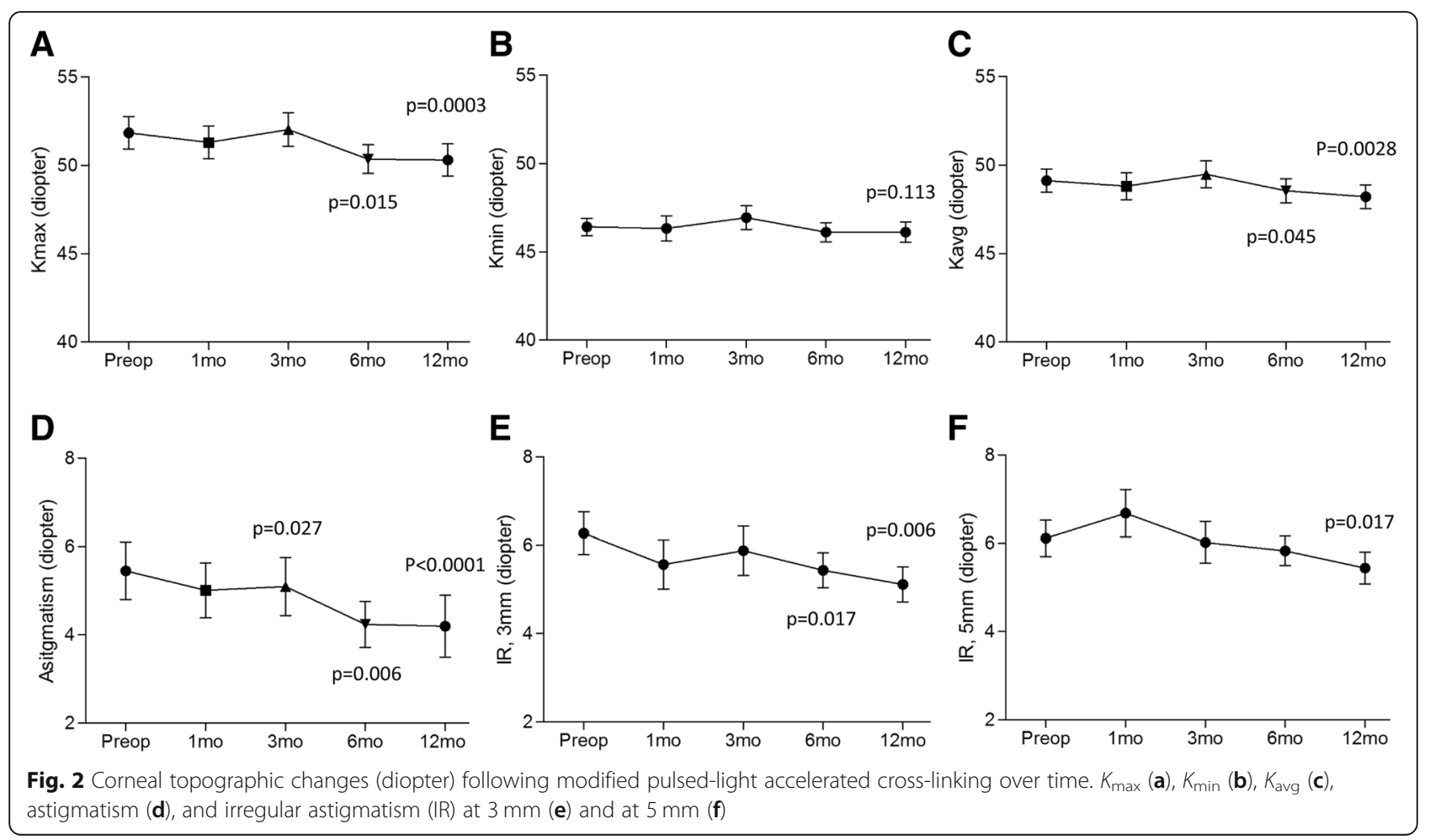




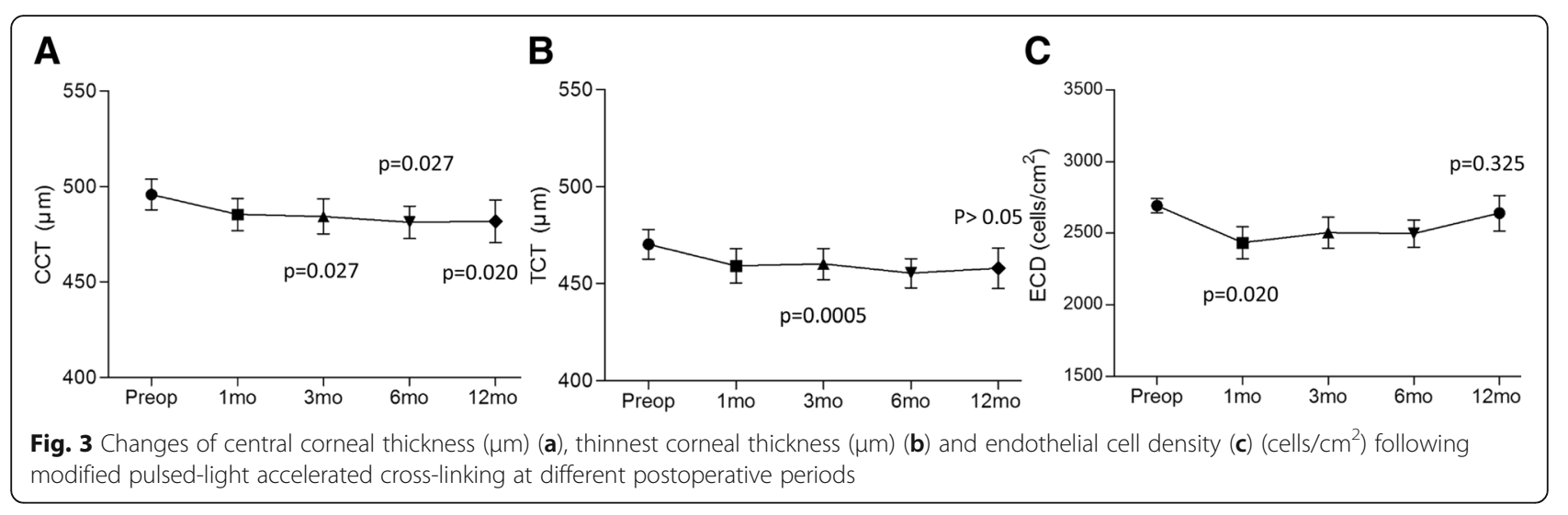

In our study, endothelial cell density was significantly reduced in postoperative 1 month compared to that pre-operatively $(p=0.02)$. It is plausible that continuous application of riboflavin may penetrate deeper microscopically than the depth that the grossly visible demarcation line indicates. This may subsequently lead to effect of UV on endothelial cells immediately post-operation. The loss of endothelial cells $(-9.61 \%)$ was not clinically significant to induce corneal edema. In addition, endothelial cell density gradually recovered and maintained up to postoperative 12 months. The overall reduction was not statistically significant $(-2.67 \%$, preop vs 12 months). It was a comparable change $\left(-91 \mathrm{cells} / \mathrm{cm}^{2}\right)$ when we compared it with previous data $\left(-54 \sim-247\right.$ cells $/ \mathrm{cm}^{2}$, Table 2$)$. Most of previous studies including the accelerated protocol demonstrated no clinically significant reduction of ECD after 12 months (Table 2) [15, 16]. Meanwhile, one study has shown a significant reduction of ECD up to 12 months after accelerated cross-linking in keratoconus [22]. An anecdotal report has presented corneal edema after cross-linking [23]. Therefore, endothelial damage should be carefully monitored when we modify these procedures. Further study is needed to explore the most appropriate continuous application time of riboflavin with the same efficacy and enhanced safety.

In this study, two patients had corneal cross-linking bilaterally. These procedures were performed sequentially without simultaneous application. It was not an intentional intra-patient control because this was a retrospective study. In fact, we conducted cross-linking in one eye in each patient, and cross-linking improved visual acuity significantly. Consequently, these patients were very satisfied with the surgical outcome and wanted to undergo cross-linking for the other eye. Subsequently, they underwent cross-linking for the contralateral eyes.

This study was limited because it did not include a direct control group with Dresden protocol. In addition, the sample size was small. Instead, we compared the efficacy and safety of our modified protocol with those of the Dresden protocol described in previous reports
(Table 2). They showed comparable outcomes. This is the first report on a novel method with clinically significant one-year follow-up outcomes.

\section{Conclusion}

Retention ring-assisted continuous application of riboflavin for 10 minutes in pulsed-light accelerated cross-linking is a comparably effective and safe treatment for halting the progression of keratoconus in 12 months when compared to outcomes of the standard Dresden protocol described in previous reports.

\section{Additional files}

Additional file 1: Supplementary Figure S1. Riboflavin is contained inside a retention ring during the application for 10 minutes $(\mathbf{A})$. A trephined silicone hydrogel bandage contact lens is applied during UV irradiation (B, C) (TIF 9431 kb)

Additional file 2: Supplementary Figure S2. Slit-lamp biomicroscopy exam after a month of modified pulsed-light accelerated cross-linking demonstrated a demarcation line ( $\mathbf{A}$, yellow arrow). Anterior segment optical coherence tomography-assisted measurement of demarcation line depth (B, yellow arrow) (TIF $6419 \mathrm{~kb}$ )

\section{Abbreviations}

AS-OCT: antesrior segment optical coherence tomography; BCVA: best corrected visual acuity; CCT: central corneal thickness; CXL: cross-linking; D: diopter; DDL: depth of demarcation line; ECD: endothelial cell density; FU: follow up; IR: irregular astigmatism; Kavg: average keratometric value; Kmax: maximal keratometric value; Kmin: minimal keratometric value; LogMAR: logarithm of minimal angle of resolution; POD: postoperative date; SD: standard deviation; SE: spherical equivalent; TCT: thinnest corneal thickness; UCVA: uncorrected visual acuity; UV: ultraviolet

\section{Acknowledgements}

None

\section{Funding}

This research received no specific grant from any funding agency in the public, commercial, or not-for-profit sectors.

\section{Availability of data and materials}

The datasets used and/or analysed during the current study are available from the corresponding author on reasonable request. 


\section{Authors' contributions}

CYK collected and analyzed the data, wrote the manuscript, and gave final approval of the manuscript to publish. MKK designed the study, wrote the manuscript, and gave final approval of the manuscript to publish.

\section{Ethics approval and consent to participate}

This retrospective study adhered to the tenets of the Declaration of Helsink and was approved by the Institutional Review Board of Seoul National University Hospital (IRB No. 1807-139-961). The need for consent was waived by Institutional Review Board of Seoul National University College of Medicine. The administrative permission was required to access the raw data from Seoul National University Hospital and this permission was also granted by Institutional Review Board of Seoul National University College of Medicine (IRB No. 1807-139-961, Seoul, South Korea).

\section{Consent for publication}

Not applicable.

\section{Competing interests}

The authors declare that they have no competing interests.

\section{Publisher's Note}

Springer Nature remains neutral with regard to jurisdictional claims in published maps and institutional affiliations.

Received: 12 November 2018 Accepted: 6 March 2019 Published online: 11 March 2019

\section{References}

1. Parker JS, van Dijk K, Melles GR. Treatment options for advanced keratoconus: a review. Surv Ophthalmol. 2015;60(5):459-80.

2. Spoerl E, Huhle M, Seiler T. Induction of cross-links in corneal tissue. Exp Eye Res. 1998;66(1):97-103.

3. Sharif $\mathrm{R}$, Fowler B, Karamichos D. Collagen cross-linking impact on keratoconus extracellular matrix. PLoS One. 2018:13(7):e0200704.

4. Wollensak G, Spoerl E, Seiler T. Stress-strain measurements of human and porcine corneas after riboflavin-ultraviolet-A-induced cross-linking. J Cataract Refract Surg. 2003;29(9):1780-5.

5. Wollensak G, Spoerl E, Seiler T. Riboflavin/ultraviolet-a-induced collagen crosslinking for the treatment of keratoconus. Am J Ophthalmol. 2003 135(5):620-7.

6. Raiskup F, Theuring A, Pillunat LE, Spoerl E. Corneal collagen crosslinking with riboflavin and ultraviolet-a light in progressive keratoconus: ten-year results. J Cataract Refract Surg. 2015;41(1):41-6.

7. Brindley GS. The Bunsen-roscoe law for the human eye at very short durations. J Physiol. 1952;118(1):135-9.

8. Shetty R, Pahuja NK, Nuijts RM, Ajani A, Jayadev C, Sharma C, Nagaraja H. Current protocols of corneal collagen cross-linking: visual, refractive, and tomographic outcomes. Am J Ophthalmol. 2015;160(2):243-9.

9. Woo JH, lyer JV, Lim L, Hla MH, Mehta JS, Chan CM, Tan DT. Conventional versus accelerated collagen cross-linking for keratoconus: a comparison of visual, refractive, topographic and biomechanical outcomes. Open Ophthalmol J. 2017;11:262-72.

10. Males JJ, Viswanathan D. Comparative study of long-term outcomes of accelerated and conventional collagen crosslinking for progressive keratoconus. Eye (Lond). 2018;32(1):32-8.

11. Vounotrypidis $E$, Athanasiou A, Kortum K, Kook D, Shajari M, Priglinger $S$, Mayer WJ. Long-term database analysis of conventional and accelerated crosslinked keratoconic mid-European eyes. Graefes Arch Clin Exp Ophthalmol. 2018;256(6):1165-72.

12. Choi M, Kim J, Kim EK, Seo KY, Kim TI. Comparison of the conventional Dresden protocol and accelerated protocol with higher ultraviolet intensity in corneal collagen cross-linking for keratoconus. Cornea. 2017;36(5):523-9.

13. Chow W, Chan TC, Yu M, Wong WW, Jhanji V. One-year outcomes of conventional and accelerated collagen crosslinking in progressive keratoconus. Sci Rep. 2015;5:14425.

14. Kymionis GD, Kontadakis GA, Hashemi KK. Accelerated versus conventional corneal crosslinking for refractive instability: an update. Curr Opin Ophthalmol. 2017;28(4):343-7.

15. Liu Y, Liu Y, Zhang YN, Li AP, Zhang J, Liang QF, Jie Y, Pan ZQ. Systematic review and meta-analysis comparing modified cross-linking and standard cross-linking for progressive keratoconus. Int J Ophthalmol. 2017;10(9):1419-29.

16. Shajari M, Kolb CM, Agha B, Steinwender G, Muller M, Herrmann E, Schmack I, Mayer WJ, Kohnen T. Comparison of standard and accelerated corneal cross-linking for the treatment of keratoconus: a meta-analysis. Acta Ophthalmol. 2018;97(1):e22-35.

17. Kamaev P, Friedman MD, Sherr E, Muller D. Photochemical kinetics of corneal cross-linking with riboflavin. Invest Ophthalmol Vis Sci. 2012;53(4):2360-7.

18. Mazzotta C, Traversi C, Paradiso AL, Latronico ME, Rechichi M. Pulsed light accelerated crosslinking versus continuous light accelerated crosslinking: one-year results. J Ophthalmol. 2014;2014:604731.

19. Jiang LZ, Jiang W, Qiu SY. Conventional vs. pulsed-light accelerated corneal collagen cross-linking for the treatment of progressive keratoconus: 12month results from a prospective study. Experimental and therapeutic medicine. 2017;14(5):4238-44.

20. Mazzotta C, Baiocchi S, Bagaglia SA, Fruschelli M, Meduri A, Rechichi M. Accelerated $15 \mathrm{~mW}$ pulsed-light crosslinking to treat progressive keratoconus: two-year clinical results. J Cataract Refract Surg. 2017;43(8):1081-8.

21. Lee HK, Ryu JS, Jeong HJ, Kim MK, Oh JY. Protection of corneal limbus from riboflavin prevents epithelial stem cell loss after collagen cross-linking. J Ophthalmol. 2018;2018:6854298.

22. Moore JE, Atkinson SD, Azar DT, Worthington J, Downes CS, Courtney DG, Moore CB. Protection of corneal epithelial stem cells prevents ultraviolet a damage during corneal collagen cross-linking treatment for keratoconus. Br J Ophthalmol. 2014;98(2):270-4.

23. Gokhale NS. Corneal endothelial damage after collagen cross-linking treatment. Cornea. 2011;30(12):1495-8.

24. Bouheraoua N, Jouve L, El Sanharawi M, Sandali O, Temstet C, Loriaut P, Basli E, Borderie V, Laroche L. Optical coherence tomography and confocal microscopy following three different protocols of corneal collagencrosslinking in keratoconus. Invest Ophthalmol Vis Sci. 2014;55(11):7601-9.

25. Brittingham S, Tappeiner C, Frueh BE. Corneal cross-linking in keratoconus using the standard and rapid treatment protocol: differences in demarcation line and 12-month outcomes. Invest Ophthalmol Vis Sci. 2014;55(12):8371-6.

26. Hagem AM, Thorsrud A, Sandvik GF, Raen M, Drolsum L. Collagen crosslinking with conventional and accelerated ultraviolet-a irradiation using riboflavin with hydroxypropyl methylcellulose. J Cataract Refract Surg. 2017; 43(4):511-7

27. $\mathrm{Ng} \mathrm{AL}$, Chan TC, Cheng AC. Conventional versus accelerated corneal collagen cross-linking in the treatment of keratoconus. Clin Exp Ophthalmol. 2016;44(1):8-14.

\section{Ready to submit your research? Choose BMC and benefit from:}

- fast, convenient online submission

- thorough peer review by experienced researchers in your field

- rapid publication on acceptance

- support for research data, including large and complex data types

- gold Open Access which fosters wider collaboration and increased citations

- maximum visibility for your research: over $100 \mathrm{M}$ website views per year

At BMC, research is always in progress.

Learn more biomedcentral.com/submissions 